\title{
A WEIERSTRASS THEOREM FOR NORMED LINEAR SPACES
}

\author{
BY P. M. PRENTER ${ }^{1}$ \\ Communicated by Ralph P. Boas, January 16, 1969
}

1. Introduction. Let $X$ and $Y$ be real normed linear spaces and let $K$ be a compact subset of $X$. Let $C(K, Y)$ denote the family of continuous functions from $K$ to $Y$ and let $\widetilde{C}(K, Y)$ denote the family of continuous functions from $X$ to $Y$ restricted to $K$. Both $C(K, Y)$ and $\tilde{C}(K, Y)$ carry the uniform norm topology given by

$$
\|f-g\|_{u}=\sup _{x \in K}\|f(x)-g(x)\| .
$$

In the event $X=Y=R$, where $R$ denotes the real numbers, the classical Weierstrass Theorem states that the family of real-valued polynomials on $X$ is dense in $C(K, Y)$ where $K=[a, b]$. If $X=R^{n}$ and $Y=R$, an application of the Stone-Weierstrass Theorem states that the family of polynomials in $n$ real variables, $n=1,2, \cdots$, is dense in $C(K, Y)$. Various generalizations of the Stone-Weierstrass Theorem have been given, notably by Hewitt, Hewitt and Zuckerman, de Branges, Kelley, and Buck. Buck's paper is notable in that he gives some attention to polynomial approximation to functions whose range is finite dimensional. The extension of the Weierstrass theory to a real separable Hilbert space setting was given by the author in [4] using the polynomial operators treated by Rall [7]. In that paper [4] it was suggested how the theory could be extended to a complex separable Hilbert space.

The purpose of the present note is to consider a Weierstrass Theorem for normed linear spaces. We will restrict ourselves to a brief outline of the theory of polynomial operators and a statement of some of the main results whose proofs together with other details and applications will appear elsewhere.

2. Basic definitions. Let $X$ and $Y$ be real or complex linear spaces. For each positive integer $n$, let $X^{n}$ denote the direct product of $X$ with itself $n$ times. An $n$-linear operator $L_{n}$ from $X^{n}$ to $Y$ is a function which is linear and homogeneous in each of its arguments separately. That is,

1 Partially sponsored by the Mathematics Research Center, United States Army, Madison, Wisconsin under contract No. DA-31-124-ARO-D-462. 


$$
\begin{aligned}
L_{n}\left(x_{1}, x_{2}, \cdots,\right. & \left.x_{i}+y_{i}, \cdots, x_{n}\right) \\
& =L_{n}\left(x_{1}, \cdots, x_{i}, \cdots, x_{n}\right)+L_{n}\left(x_{1}, \cdots, y_{i}, \cdots, x_{n}\right)
\end{aligned}
$$

and $L_{n}\left(x_{1}, x_{2}, \cdots, a x_{i}, \cdots, x_{n}\right)=a L_{n}\left(x_{1}, x_{2}, \cdots, x_{i}, \cdots, x_{n}\right)$. We define a 0 -linear operator $L_{0}$ to be a constant function and we identify $L_{0}$ with its range so that $L_{0} x=L_{0}$ for all $x \in X$. The operator $P$ from $X$ to $X$ given by

$$
P x=L_{n} x^{n}+L_{n-1} x^{n-1}+\cdots+L_{1} x+L_{0}
$$

is called a polynomial operator of degree $n$ in $X$ whereas $P x$ is called a polynomial of degree $n$ in $X$. It is a simple matter to prove that $P x$ corresponds in a natural way to an ordinary polynomial of degree $n$ in $k$ variables in the event $X$ is $k$ dimensional and $Y$ is one dimensional. If $K$ is a compact subset of $X$, we let $P(K, Y)$ denote the family of continuous polynomial operators from $K$ to $Y$ where $P(K, Y)$ carries the uniform norm topology. Clearly, not all polynomials are continuous since any unbounded linear operator is a polynomial of degree one.

Part of our theory in an infinite dimensional setting makes use of linear spaces with a particular property. A normed linear space $X$ is said to have Property $M, M>0$, if for each finite dimensional subspace $Z \subset X$ and for each compact subset $K$ of $X$ there exists a continuous projection $P$ of $X$ onto $Z$ such that $\|P x\| \leqq M\|x\|$ for all $x \in K$.

Property $M$ as here defined is not to be confused with property $P$. as defined by Goodner [1], Kelley [2], and Nachbin [3]. It is clear that every Hilbert space has Property 1.

Polynomials occur frequently, the Navier-Stokes equations being an impressive example. Two simple examples are the expressions

$$
a_{2}(t) x^{\prime \prime}(t)+a_{1}(t) x^{\prime}(t)+x^{3}(t)
$$

where $a_{1}, a_{2}$, and $a_{3}$ are continuous and

$$
x(s)-\int_{0}^{1} \int_{0}^{1} \int_{0}^{1} k\left(s, t_{1}, t_{2}, t_{3}\right) x\left(t_{1}\right) x\left(t_{2}\right) x\left(t_{3}\right) d t_{1} d t_{2} d t_{3} .
$$

If we let $X=C^{2}[0,1]$ and let $Y=C[0,1]$ then (1) is a polynomial of degree three from $X$ to $Y$. If, on the other hand, we let $X=L^{2}[0,1]$ and insist that

$$
\int_{0}^{1} \int_{0}^{1} \int_{0}^{1} \int_{0}^{1}\left|k\left(s, t_{1}, t_{2}, t_{3}\right)\right|^{2} d t_{1} d t_{2} d t_{3} d s<\infty
$$

then (2) is a polynomial of degree three (a cubic equation) from $X$ into $X$. 
3. Main results.

Theorem 1. Let $X$ and $Y$ be finite dimensional, real normed linear spaces and let $K$ be a compact subset of $X$. Then $P(K, Y)$ is dense in $C(K, Y)$.

Our proof of this result depends in an essential way on the matrix representation of a polynomial operator [5].

Theorem 2. Let $X$ and $Y$ be real normed linear spaces and let $X$ have Property $M, M>0$. Then $P(K, Y)$ is dense in $\widetilde{C}(K, Y)$.

The proof of this depends on Theorem 1 and the total boundedness of $K$.

Theorem 3. Let $X$ and $Y$ be real normed linear spaces and let $K$ be a compact subset of $X$. Then, for each $\epsilon>0$ and for each $F \in \widetilde{C}(K, Y)$, there exists a finite dimensional subspace $X^{(n)}$ of $X$, a continuous function $S$ from $X$ onto $X^{(n)}$ and a continuous polynomial operator $P$ from $S[K]$ into a finite dimensional subspace $Y^{(m)}$ of $Y$ for which

$$
\|F(x)-P(S x)\|<\epsilon \quad \text { for all } x \in K \text {. }
$$

Here $S[K]=\{S(x): x \in K\}$.

The proof of Theorem 3 is similar to that of Theorem 2. It tells us that any continuous function from $X$ to $Y$ can be uniformly approximated on a compact set by a polynomial operator of finite rank on a finite dimensional compact subset $\tilde{K}=S[K]$ which has the property $\|x-S x\|<\delta$ for all $x \in K$ where $\delta>0$ and $\delta=\delta(\epsilon)$. This theorem has interesting numerical applications which will be examined in a later paper.

\section{REFERENCES}

1. D. B. Goodner, Projections in normed linear spaces, Trans. Amer. Math. Soc. 69 (1950), 89-108.

2. J. L. Kelley, Banach spaces with the extension property, Trans. Amer. Math. Soc. 72 (1952), 323-326.

3. Leopoldo Nachbin, $A$ theorem of Hahn-Banach type for linear transformations, Trans. Amer. Math. Soc. 68 (1950), 28-46.

4. P. M. Prenter, A Weierstrass Theorem for real separable Hilbert spaces, MRC Technical Report 868, April 1968.

5. - Matrix representations of polynomial operators, MRC Technical Report 929 (to appear).

6. L. B. Rall, Quadratic equations in Banach space, Rend. Circ. Mat. Palermo 10 (1961), 314-332.

7. - Solution of abstract polynomial equations, MRC Technical Report 892, August 1968.

Colorado State University, Fort Collins, Colorado 80521 